\title{
Carbon Monoxide Sensor for Combustion Feedback Control
}

\author{
Joel A. Silver ${ }^{*}$ and Shin-Juh Chen ${ }^{\dagger}$ \\ Southwest Sciences, Inc., 1570 Pacheco Street, Suite E-11, Santa Fe, NM 87505
}

\begin{abstract}
A sensor for carbon monoxide detection based on broadband absorption spectroscopy with a gas correlation filter is developed using a miniaturized light source and detector without the need of a mechanical chopper to alternate between two gas correlation cells into the optical path. Measurements were conducted using calibrated $\mathrm{CO} / \mathrm{N}_{2}$ mixtures and sampled combustion products from a diffusion flame. The detection sensitivity was found to be $100 \mathrm{ppm}$ for the measured range of 100 to $500 \mathrm{ppm}$. The sensor can be used to control individual burners in small scale combustion systems in order to maintain combustion efficiency and minimize pollutant emissions during their operation life cycle.
\end{abstract}

\section{Introduction}

Industrial combustion systems account for 75 percent of the total energy end use in the U.S. manufacturing sector. ${ }^{1}$ Fired heaters and steam systems account for 38 and 35 percent, respectively. Burners are used in these combustion systems to generate heat for a wide variety of commercial processes ranging from food production to metal fabrication. Common to many of these applications is the need to generate heat via combustion efficiently so as to minimize the product cost. Concurrently, the generation of pollutant by-products such as particulates, nitrogen oxides (in particular, $\mathrm{NO}_{2}$ ) and carbon monoxide (CO) must be minimized, as governed by regulatory agencies. In smaller industrial boilers, commonly used pollution minimization systems such as selective catalytic reduction (SCR), thermal DeNOX, staged combustion, pulsed combustion, recirculation of flue gases, baghouses and electrostatic precipitation may not be employed due to the smaller size of these boilers ( $<100 \mathrm{MBTU})$ and the relatively larger cost of implementing these strategies on the smaller systems. As a result, although the sum of pollutant levels emitted from these smaller sources is significant, the methods that can be applied to control them are limited. The simplest approach is to control fuel-air ratios, yet this is often just a one time adjustment. Another approach is to simultaneously monitor oxygen $\left(\mathrm{O}_{2}\right), \mathrm{CO}$, NOx, and carbon dioxide $\left(\mathrm{CO}_{2}\right)$, but existing instruments to perform these measurements are not cost-effective for small boilers or furnaces.

Unlike larger industrial boilers, where an array of burners may be used to heat the system, smaller industrial boilers have only one or a few burners, so that an in situ burner-based sensor is a reasonable method to use for continuously monitoring the combustion process. In many cases, a periodic or on-line flue gas oxygen measurement is performed to assess combustion performance. Unfortunately, this only provides a measure of the amount of excess air, but in no way can account for the effects of variations in fuel composition, sizing distribution (for coal), and the amount of moisture present, which can significantly affect the combustion efficiency and the amount of pollutants generated.

A low cost, active monitor/feedback control system could have an important effect on minimizing pollution from these sources. The approach here is to monitor $\mathrm{CO}$ in the individual burner exhaust and to feed back a signal to the air flow control so as to actively adjust the fuel-air ratio for optimal performance. The presence of high CO concentrations indicates incomplete combustion of fuels which results in non-optimal combustion efficiency. By monitoring CO concentrations, $\mathrm{NO}_{\mathrm{x}}$ emission levels can be indirectly controlled by optimizing the level of excess air during combustion. The relationships between $\mathrm{CO}, \mathrm{NO}_{\mathrm{x}}$ and heat loss are well known. With CO maintained between 50 and 300 parts-permillion (ppm), independent of load, the burner is in the best operating regime and $\mathrm{NO}_{\mathrm{x}}$ is still well regulated. ${ }^{2}$ If $\mathrm{CO}$ can be monitored across this 50 to $300 \mathrm{ppm}$ range in the presence of combustion gases, then a useful feedback control input

\footnotetext{
${ }^{*}$ Executive Vice President, Southwest Sciences, Inc., Santa Fe, NM

${ }^{\dagger}$ Senior Research Scientist, Southwest Sciences, Inc., Santa Fe, NM, Member
} 
exists and flame optimization can be achieved. Since accurate readings just above and below this range are needed to adjust feedback controls, the required sensitivity of the sensor is defined to be $10 \mathrm{ppm}$ of CO.

A variety of laser-based gas absorption, chemiluminescence, and non-dispersive infrared (NDIR) methods have been studied and in some cases implemented to monitor oxygen, carbon dioxide, carbon monoxide, water, methane, $\mathrm{NO}_{\mathrm{x}}$ and other gases. Laser-based flue and stack sensors are becoming established for pollution measurements ${ }^{3,4}$ and traditional non-dispersive infrared (NDIR), electrochemical cells, solid state sensors and thermocouples have all been used with varying degrees of success. However, a CO sensor to directly monitor burners of small boilers and furnaces must be low cost, rugged, compact, highly sensitive (low ppm levels), fast response (a few seconds), and unaffected by high concentrations of water vapor and carbon dioxide. This paper discusses the development and testing of such a sensor system to meet the challenge of monitoring each burner of small industrial combustion systems.

\section{Detection of Carbon Monoxide}

\section{A. Broadband Absorption Spectroscopy}

The major consideration in designing an optical-based detection sensor for post-combustion CO is the complexity of the flue gas mix. A typical process burner output operating near optimal efficiency contains several chemical species in varying concentrations. Table 1 show a list of typical chemical species and their range of concentration in the burner flue gases.

Table 1: Typical Chemical Species in Burner Flue Gas

\begin{tabular}{|l|c|}
\hline Chemical Species & Concentration (vol. basis) \\
\hline Oxygen & $0-5$ percent \\
\hline Hydrocarbon Gases & $0-1$ percent \\
\hline Carbon Dioxide & $9-10$ percent \\
\hline Water Vapor & $18-19$ percent \\
\hline Carbon Monoxide & $10-100,000 \mathrm{ppm}$ \\
\hline Nitrogen Oxides & $10-200$ ppm \\
\hline Nitrogen & 70 percent (balance) \\
\hline
\end{tabular}

Optical techniques for measuring $\mathrm{CO}$ can be sensitive and highly selective, but in the presence of the relative gas concentrations above, problems may arise. In essence, there are two optical approaches for detecting CO, using spectrally-narrowband lasers (primarily diode lasers) and non-dispersive infrared (broadband) systems. Preference for the NDIR-based methods is discussed below.

Using the HITRAN and HITEMP databases, ${ }^{5}$ spectra for flame exhaust gas at different optical wavelengths and temperatures are readily computed. For detection of carbon monoxide, there are three viable absorption bands - near $1500 \mathrm{~nm}$ and $2300 \mathrm{~nm}$ (near-infrared), and $4650 \mathrm{~nm}$ (mid-infrared). In each of these spectral regions, some of the other gases present absorb light as well. Where the amount of light absorbed is relatively small, the fraction of incident light intensity transmitted through a cell of absorbing gas is determined from Beer's law,

$$
\frac{I_{0}-I}{I_{0}}=\alpha=\sigma(v) N \ell,
$$

where $I_{0}$ is the incident light intensity on the gas, $I$ is the amount of transmitted light and $\alpha$ is the fractional amount of 
light absorbed for $\alpha<<1$. This fractional absorbance is equal to the product of the absorption cross section $\sigma(v)$, the number density of the gas $N$ and the path length of light through the gas $\ell$. The cross section is wavelength dependent and is usually much larger for the fundamental vibrational transitions in the mid-infrared than for the overtone bands in the near infrared.

Detailed calculations for these three bands at temperatures between $296 \mathrm{~K}$ and $500 \mathrm{~K}$ show that only the infrared band at $4650 \mathrm{~nm}$ has $\mathrm{CO}$ lines that absorb at levels comparable to water and carbon dioxide lines in that region (using the expected concentration levels listed in Table 1). Near $1550 \mathrm{~nm}, \mathrm{CO}_{2}$ and $\mathrm{H}_{2} \mathrm{O}$ absorptions are much larger than for $\mathrm{CO}$ and at $2300 \mathrm{~nm}, \mathrm{CO}$ is overwhelmed by methane (or possibly other hydrocarbons) and water. In addition, neither of these bands has sufficient absorption cross sections, so that a short $(10 \mathrm{~cm})$ optical path is not feasible without using a multiple pass optical cell.

Based on the HITRAN spectral database, the fundamental vibrational band of $\mathrm{CO}$ at $4650 \mathrm{~nm}$ near room temperature is very strong and has absorbances comparable to those of water and carbon dioxide near $4650 \mathrm{~nm}$. Figure 1 illustrates the expected spectrum for a flue gas mixture cooled to near room temperature for a $10-\mathrm{cm}$ sample cell containing $50 \mathrm{ppm}$

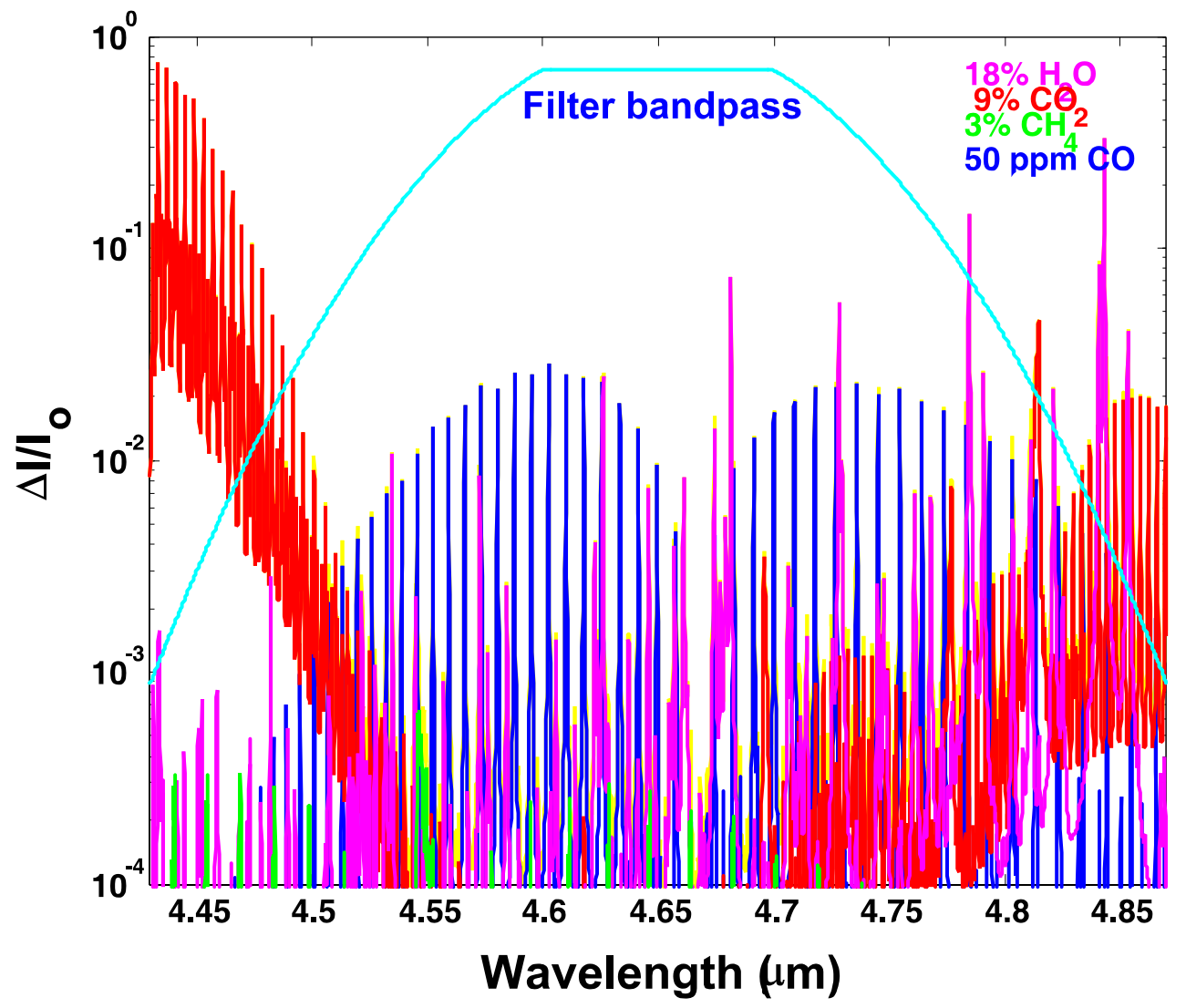

Figure 1 - Absorption spectra for burner flue gases using a 10-cm optical path length. Flue gases include $18 \% \mathrm{H}_{2} \mathrm{O}, 9 \% \mathrm{CO}_{2}, 3 \% \mathrm{CH}_{4}$, and $50 \mathrm{ppm} \mathrm{CO}$.

of CO. The clean separation of some CO lines from the rest of the spectrum might invite one to think that a laser-based absorption method would be best. While possibly true, multiple factors cause us to reject this assumption. The only lasers that exist for infrared sensing are lead salt diode lasers (which require liquid nitrogen or liquid helium cooling) and quantum cascade (QC) lasers, which can operate either with liquid nitrogen or with a thermoelectric cooler nominally near room temperature. While this latter choice seems promising, the reality is that a QC system costs about $\$ 20,000$. Room temperature operation requires complex pulsed, high current sources and operational QC lasers in this particular wavelength range have just recently been reported at the research stage with pulsed, cooled operation. Detection of CO 
using NDIR-based systems is a more cost-effective method and is described in the following sections.

\section{B. Conventional Non-Dispersive Infrared Detection (NDIR)}

For conventional non-dispersive infrared detection, a broadband spectral source (such as a glowbar or lamp) is imaged through the sample gas and then through a narrowband interference filter which matches the spectrum of the gas of interest. For example as illustrated in Fig. 1, a typical filter bandpass function as well as a stick figure spectrum of $\mathrm{CO}$ at $296 \mathrm{~K}$ near $4650 \mathrm{~nm}$ are shown. The filter response is selected to match the absorption spectrum of the gas of interest and reject all other wavelengths (presumably where other gases absorb strongly). Applying this infrared filter to the $\mathrm{CO}$ spectrum (with $10 \mathrm{ppm}$ of $\mathrm{CO}$ ) to limit the transmission band, only about $16 \%$ of the total absorption is due to $\mathrm{CO}$, the balance of absorbed light is mostly by water vapor.

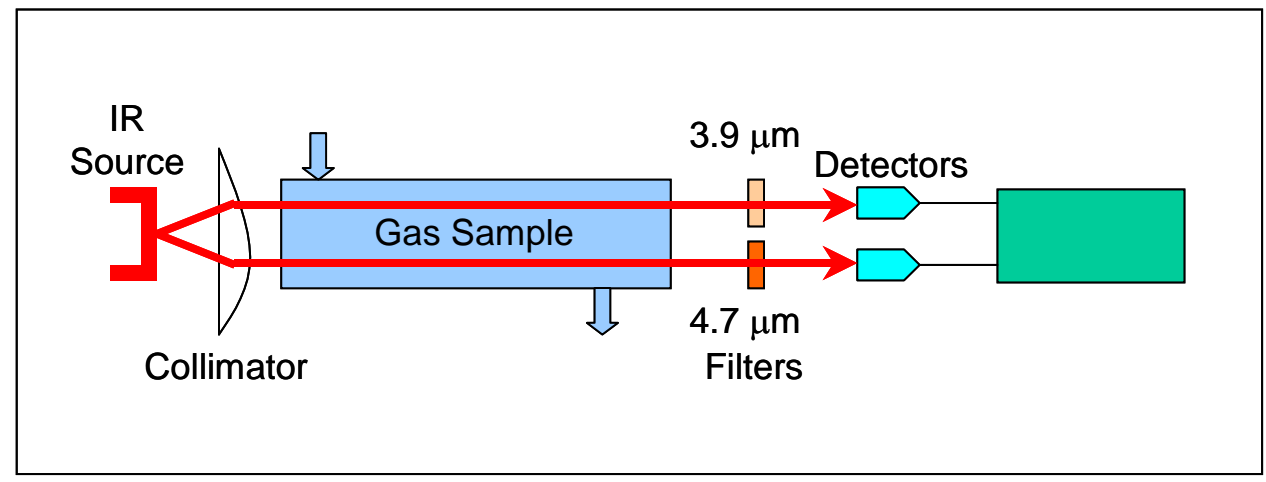

Figure 2. Conventional NDIR-based detector.

The conventional NDIR instrument operates by comparing the total amount of light transmitted at this filtered wavelength band as compared to that of another wavelength band (typically $3900 \mathrm{~nm}$ ), where no CO absorbs. A schematic of such a sensor is shown in Fig. 2. The difference in absorption between the two bands is used to determine the concentration of CO present. This approach makes the simplistic assumption that all absorption in this $4650 \mathrm{~nm}$ band is from the target gas, CO. Clearly, this assumption is unsuitable here since, as noted above, most of the absorbance within the band near $4650 \mathrm{~nm}$ originates from gases other than CO. Since the spectrum is congested with absorption lines other than CO, conventional NDIR sensing (broadband IR light source plus bandpass filter) is insufficient to selectively detect CO. Combining NDIR with a gas correlation filter (GCF) is a common solution to overcome this spectral overlap problems.

\section{Non-Dispersive Infrared Detection with Gas Correlation Filter (NDIR/GCF)}

Combining NDIR with a gas correlation filter (GCF) overcomes the spectral overlap problem and permits much

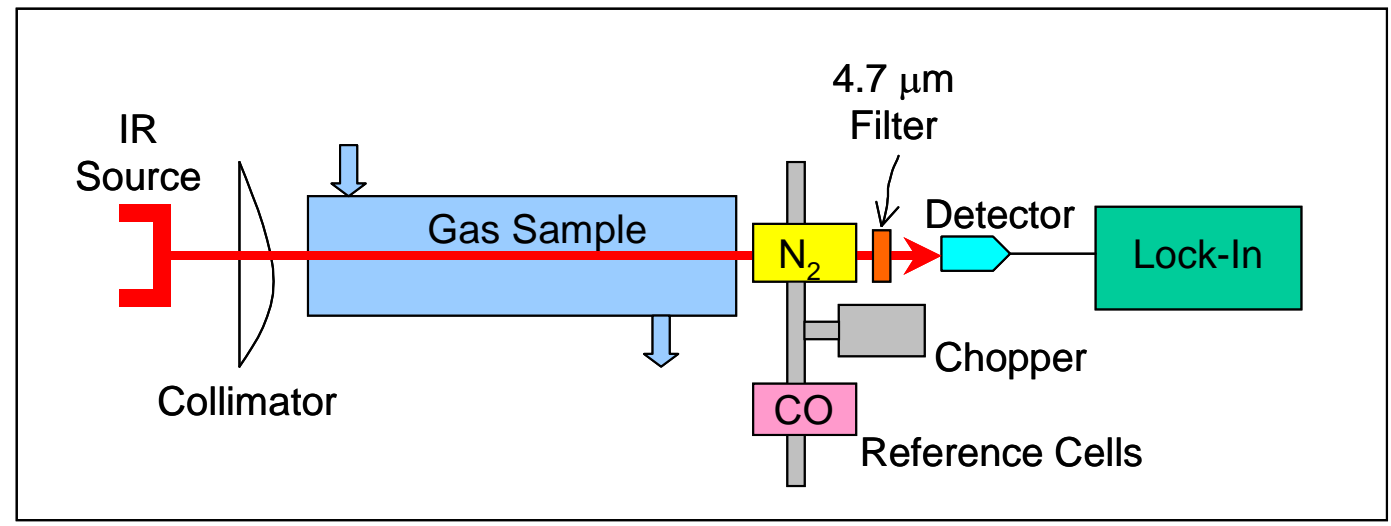

Figure 3. NDIR-GCF Detector. 
higher detection sensitivities when all of the absorption is not due only to CO. A mechanical chopper wheel is used to alternate two gas correlation cells into the optical absorption path, as shown in Fig. 3. One cell contains a large amount of the gas of interest (in this case, $\mathrm{CO}$ ) and the second has a non-absorbing "blank" gas such as nitrogen. Using phase sensitive detection, the difference in light transmitted to the detector between these two cases depends only on the difference in CO concentration between the two paths. By initially calibrating the system when the sample path is empty, then the observed output signal is proportional to the difference in the cell concentrations, from which the sample concentration can be realized. All other gases absorb equally for either path and show no difference signal. This approach has been the basis for a number of commercial instruments. For the case here, $10 \mathrm{ppm}$ of CO contributes to about 1/6 of the total absorption, so that a dynamic range of 10 to $300 \mathrm{ppm}$ CO would be easily obtained despite the other gases present. The downside of this approach is that it requires a rugged mechanical chopper to rotate the gas cells. These instruments are large and expensive.

\section{Micromirror-Based Non-Dispersive Infrared Detection (MM-NDIR)}

A major simplification of the NDIR-GCF sensor is to remove the mechanical chopper and use a smaller, less powerhungry light source. This not only reduces complexity of the instrument, but also cost. This is accomplished by incorporating three new components: (a) a compact, low power, miniature, infrared light source, (b) a MEMS mirror to alternate the light beam between both paths, and (c) a newly available infrared detector that is smaller and simpler to implement. Each component is described below, and a schematic of the NDIR system incorporating the MEMS mirror (MM-NDIR) is illustrated in Fig. 4.

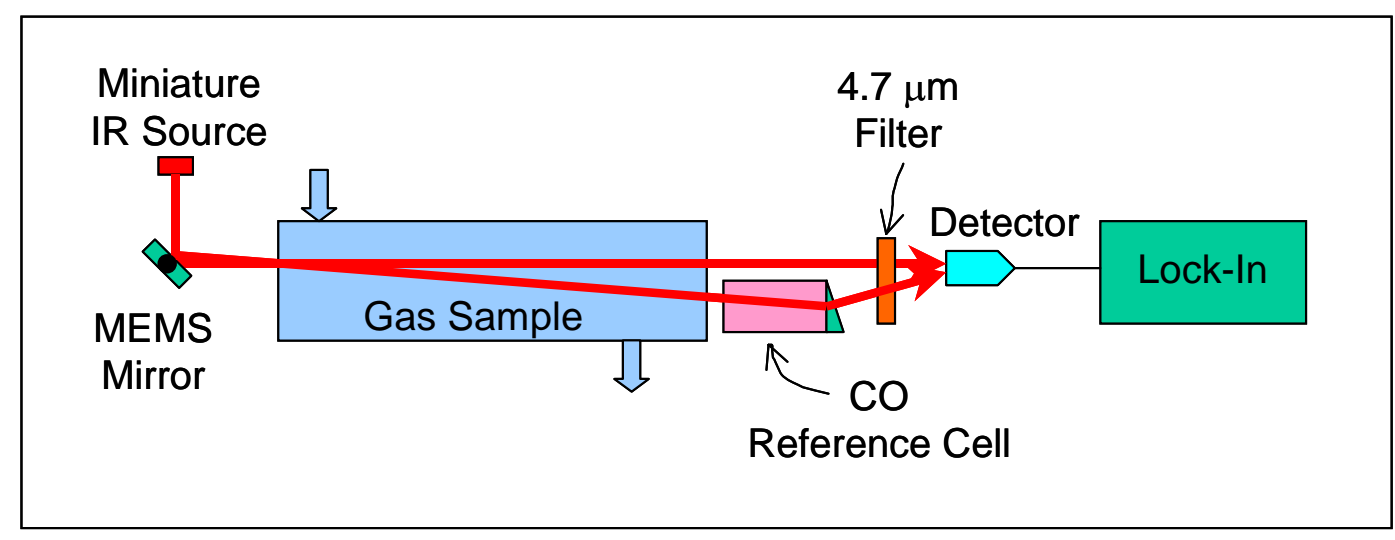

Figure 4. MM-NDIR Detector.

The IR source (IR-50, HawkEye Technologies) has an aperture of 0.9 $\mathrm{cm}$, and $0.3 \mathrm{~cm}$ thick (excluding the mounting pins). It has a nominal power output of about $7.6 \mathrm{~mW}$ (computed using a tungsten emitter of 1$\mathrm{mm}^{2}$ at $750^{\circ} \mathrm{C}$ ). The input power is about $0.9 \mathrm{~W}$ at $6.7 \mathrm{~V}$. An evaluation kit MEMS system provided by Applied MEMs Inc, but no longer commercially available, is used. This is a $3 \mathrm{~mm} \times 3 \mathrm{~mm}$ square gold-coated mirror that can be moved over the $\mathrm{X}$-axis over a $\pm 1^{\circ}$ range at $670 \mathrm{~Hz}$ and the $\mathrm{Y}$-axis over a $\pm 12^{\circ}$ range at $50 \mathrm{~Hz}$. For our application, the $\mathrm{Y}$ axis provides plenty of angular range at a reasonable speed. A photograph of the MEMS mirror is shown in Fig. 5. The advantage of the MEMs approach is that the expected reliability is very high and that the size and power draw for this is much lower than for the mechanical chopper wheel. Finally, most infrared detectors require thermoelectric cooling and/or need to operate as ac-coupled devices. A new HgCdZnTe detector (PV-5, VIGO Systems) which operates in dc-coupled, photovoltaic mode is selected. The detector can be thermoelectrically cooled to provide higher sensitivity. The electronics for this MM-NDIR system are quite simple. A square wave

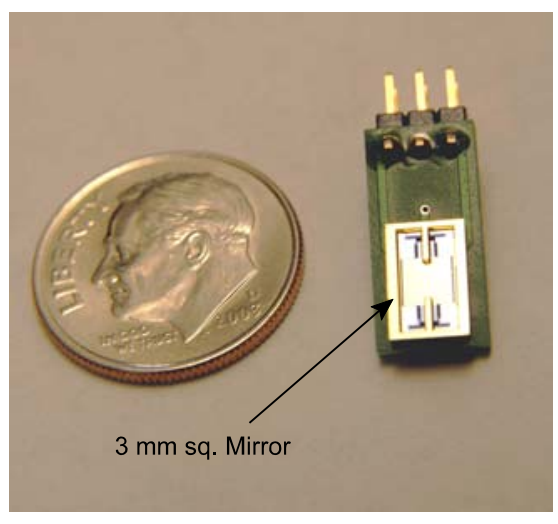

Figure 5. MEMS mirror. 
drive the MEMS mirror position, and signal analysis from the photodetector requires only a phase-sensitive detector chip. Scaling for span and offset, the system outputs a voltage based on the CO concentration for combustion control purposes. The entire sensor runs at 5 volts, and draws a total power of less than $5 \mathrm{~W}$.

\section{Development and Testing of a MM-NDIR CO Sensor}

\section{A. Original Optical Setup}

The initial MM-NDIR sensor is shown in Fig. 6. The optical layout is made compact by use of off-axis paraboloidal (OAP) reflectors. Here the light from the source is focused onto the MEMS mirror, which is located at the focal point of the OAP, so that all reflected rays are parallel. The MEMS mirror alternates the beam between the two reference cells, through the sample cell (inner dimensions of $10 \mathrm{~cm}$ long and $2 \mathrm{~cm}$ dia.) and onto a second OAP that refocuses both paths onto a single HgCdZnTe detector. A miniature diaphragm pump (Thomas, $900 \mathrm{cc} / \mathrm{min}$ Max. Flow, $50 \mathrm{~mA}, 6 \mathrm{~V}$ ) pulls air samples through the sample cell. An infrared bandpass filter is used to isolate only the spectral region of interest. These two cells are fixed and 7-cm long; one is filled with pure nitrogen and the other with pure CO. The mirror is dithered at frequencies between 10 and $100 \mathrm{~Hz}$. The detector output is fed into a preamplifier and then into a lock-in. Initial alignment measurements revealed some problems. The light source was fairly weak and we had a great deal of difficulty getting sufficient intensity on the detector. Much of this is due to the problem of trying to image an extended source $\left(1.5 \mathrm{~mm}^{2}\right)$ over tens of $\mathrm{cm}$ of path. Originally, much of the problem was thought to be trying to position the MEMs mirror at two foci simultaneously (source and OAP). In addition, the HgCdZnTe detector showed time response problems, which could only be characterized as bizarre. Given the time constraints of this project, the defective detector was replaced with a InSb detector. Moreover, from the intensity measurements and by observing the shape of the detected waveform on an oscilloscope, the MEMs mirror was not providing a good square wave. It seemed to respond in a more damped fashion even at low $(10 \mathrm{~Hz})$ frequencies. We also had a problem getting the amplitude to stabilize at any frequency. At first we thought that our mounting of the MEMs circuit board was poor, but subsequent study showed that the mirror exhibited thermal drifts as well. The optical setup was redesigned and some components were replaced to mitigate the problems found in the original setup.

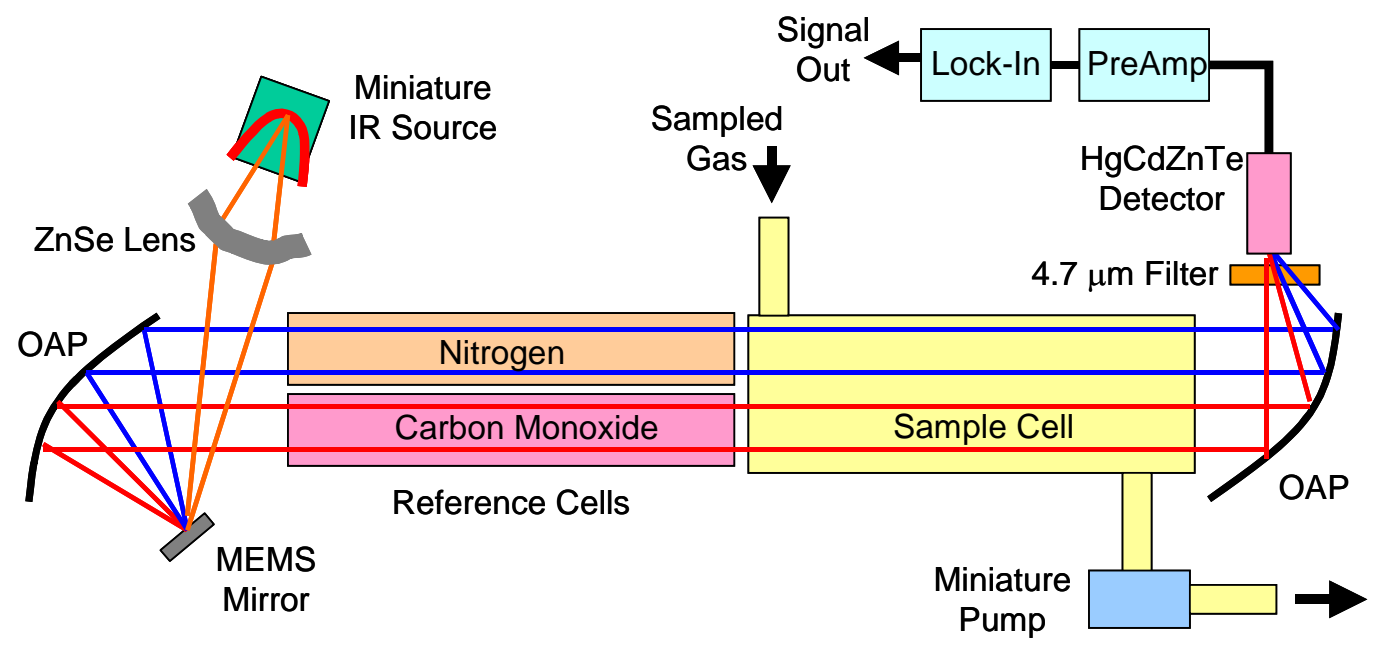

Figure 6. Original Optical Setup of MM-NDIR CO Sensor.

\section{B. Revised Optical Setup}

In the revised configuration as illustrated in Fig. 7, a pinhole aperture (600 $\mu$ m diameter) is included to spatially filter the beam for better imaging. This pinhole was probably still too large, but was needed to get sufficient signal at the detector. The nitrogen cell is not needed for this particular application, since in room air the amounts of spectrally interfering gases $\left(\mathrm{H}_{2} \mathrm{O}\right.$ and $\left.\mathrm{CO}_{2}\right)$ were insignificant relative to their absorbances in the post-combustion sample cell where 


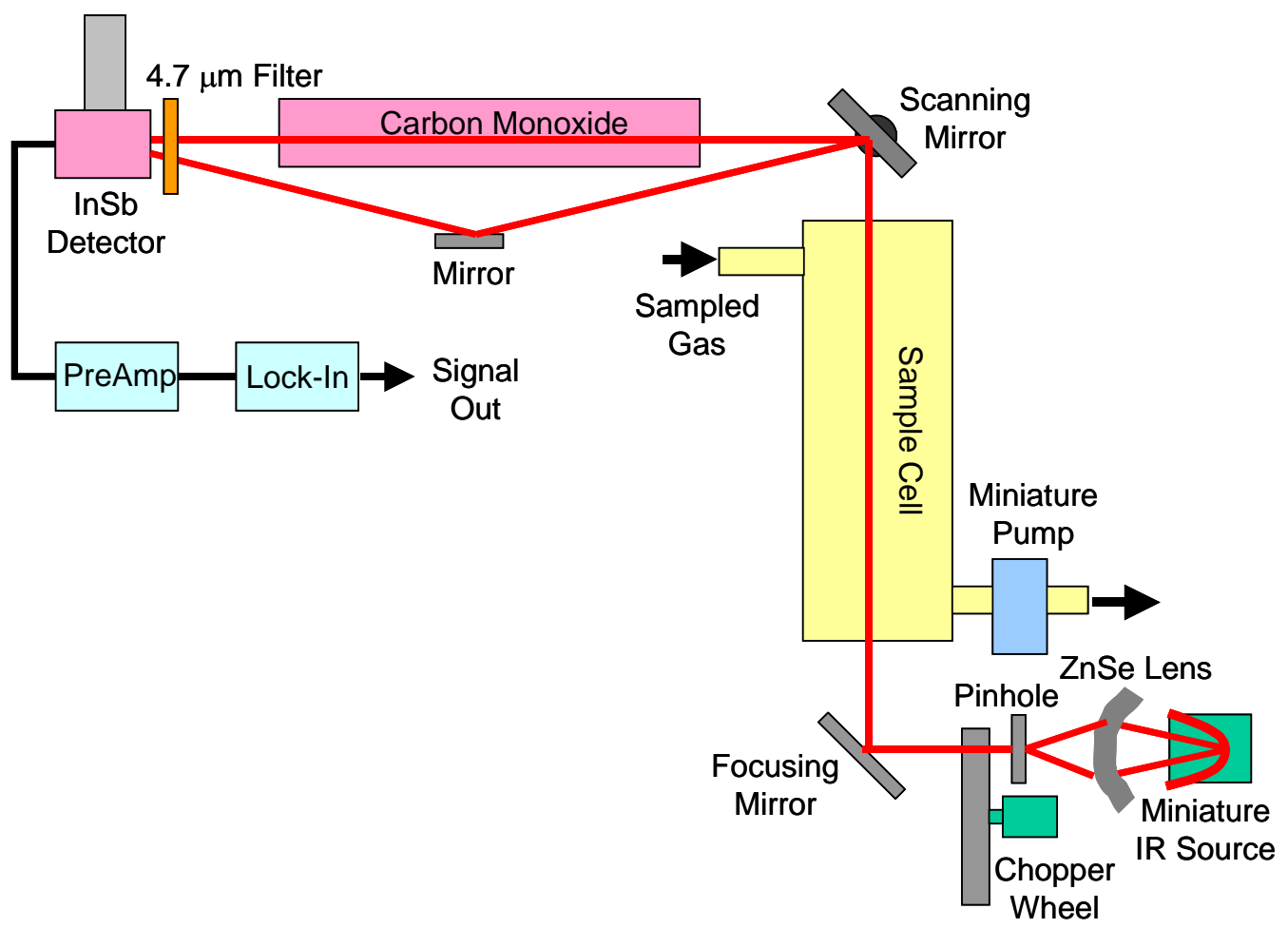

Figure 7. Revised Optical Setup of MM-NDIR CO Sensor.

they would be at much higher concentrations ( 18 and 9 percent, respectively). The design is somewhat simplified since only the CO reference cell is needed. In retrospect, the original optical design (see Fig. 6) is probably still better and the lack of intensity is more likely just the need for better spatial filtering.

A readily available liquid nitrogen cooled InSb detector $\left(D^{*}=1 \times 10^{11} \mathrm{~cm} \cdot \mathrm{Hz}^{1 / 2} / \mathrm{W}\right)$ is used and worked just fine. The original detectivity of the room-temperature HgCdZnTe photodiode was probably too low in any case $\left(\mathrm{D}^{*} \sim 3 \times 10^{8}\right.$ $\mathrm{cm} \cdot \mathrm{Hz} / / 2 / \mathrm{W}$ ) and that future sensors should require a thermoelectrically cooled detector $\left(\mathrm{D}^{*} \sim 2 \times 10^{10} \mathrm{~cm} \cdot \mathrm{Hz}^{1 / 2} / \mathrm{W}\right)$. Any drifts in the absolute intensity on the detector (whether arising from alignment or source power variations) must be accounted for. If the dc-coupled detector ( $\mathrm{HgCdZnTe}$ ) were working, this would be easily accomplished by monitoring the total intensity at the detector. Since the InSb detector is ac-coupled, a $1.05 \mathrm{kHz}$ chopper is added in front of the pinhole and a second lock-in is used to monitor the total light intensity. The MEMs mirror was replaced with a voltagecontrolled micro-scanner mirror, which worked perfectly. The mirror size is about $5 \mathrm{~mm}$ and was scanned at $40 \mathrm{~Hz}$ with a square wave.

\section{CO Measurements at Room Temperature}

A calibrated CO/ $\mathrm{N}_{2}$ mixture (500 ppm CO, $\mathrm{N}_{2}$ balance) was further diluted with $\mathrm{N}_{2}$ to provide $\mathrm{CO} / \mathrm{N}_{2}$ mixtures with $\mathrm{CO}$ concentrations ranging from 100 to $500 \mathrm{ppm}$. The methodology used to determine the amount of CO in the sample cell is as follows. The sample cell (see Fig. 7) is initially filled with pure CO to remove any light intensity difference between the two light paths. The offset feature of the lock-in is used to define this value as zero. In doing this then, the relative lock-in signal when $\mathrm{CO}$ is present in the sample cell is proportional to absolute CO concentration. With the sample cell empty (pure $\mathrm{N}_{2}$ ), the differential signal at the lock-in measures the differences in absorption between the nitrogen and CO reference cells, which is only the amount of light absorbed by the pure CO cell, as well as differences in total intensity in the two legs that reach the detector.

After normalizing for intensity, we now measured GCF signals versus calibrated amounts of CO in nitrogen. An example of these measurements is shown in Fig. 8. Each measurement is averaged over 60 seconds. The sensitivity of the calibration curve was poorer than expected, with a detection sensitivity of about $100 \mathrm{ppm}$ instead of the desired 10 ppm. 
The poor performance of the prototype CO sensor could be attributed to two factors: (1) non-optimal imaging of the light source, and (2) improper reference cell concentration. The imaging issue was addressed by measuring the detector intensity at various positions in the optical path. Starting with a measured integrated output of the lamp at $7.6 \mathrm{~mW}$ (exactly as expected for a tungsten emitter of $1-\mathrm{mm}^{2}$ area at $750^{\circ} \mathrm{C}$ ), we lose at least $10^{5}$ of the initial intensity through the entire system. Only $0.4 \%$ of the light passes through the bandpass filter, less than would be expected from a blackbody source at $750^{\circ} \mathrm{C}$. The window on the lamp must be starting to cut off at longer wavelengths, so a slightly different version of the lamp will be used in future prototype. In addition, losses of $35 \%$ occur at each of the four sapphire window surfaces with an additional $20 \%$ loss in the lens. Even with a relatively large $1 \mathrm{~mm}$ spatial filter, probably less than half of the source intensity is passed. Combining these factors results in an imaging loss over the long $45 \mathrm{~cm}$ path of better than a factor of 30 . Clearly the overall path must be much shorter, with a smaller diameter spatial filter to allow better imaging. The tradeoff of transmission through the pinhole will be more than offset by a shorter optical path and better imaging onto the detector.

The second issue is the integrated absorbance of the reference cell. The idea of a GCF is to selectively absorb light where the CO lines lie, but not elsewhere. The current design was too long $(7 \mathrm{~cm})$ with pure $\mathrm{CO}$, and the Lorentzian tails between lines add up so that light at all wavelengths in the filter pass band were significantly absorbed by the cell, thus, lowering its dynamic range and sensor sensitivity. A better design would be a more compact, 1-cm diameter cell with only $20 \%$ CO. One could also improve overall transmission by attaching this reference cell directly to the sample cell window, so that only three total IR windows are used. These windows could also use other materials/coatings to further reduce losses.

\section{CO Measurements in a Diffusion Flame}

An 8-cm long methane-air diffusion (Wolfhard-Parker) burner was set up for sampling using $0.3 \mathrm{~cm}$ diameter stainless steel tubing attached to Tygon tubing. Using the miniature diaphragm pump, a small flow of combustion gases was sampled at various positions in the flame. The pump has a maximum flow rate of $900 \mathrm{cc} / \mathrm{min}$, which gives a maximum of 30 air exchanges per minute in the 30-cc sample cell. As expected, strong signals were observed near the peak flame position on the fuel-rich side where $\mathrm{CO}$ is expected to reach percent levels. Further into the fuel side, concentrations dropped off to a few hundred ppm. No CO was observed deep into the air side of the flame. One important observation of these measurements is that since the needed gas flow is so small, its temperature rapidly equilibrated to near room temperature only a few cm away from the flame. Thus all sampling in the sensor would always be near $300 \mathrm{~K}$, independent of the flame conditions.

\section{Summary}

A low cost CO sensor for feedback control systems that can be used with individual burners is developed and tested. This MM-NDIR CO sensor uses miniaturized light sources and detectors with broadband gas correlation optical absorption to make measurements of carbon monoxide, which can be used to regulate combustion fuel-air ratios. The detector sensitivity was found to be $100 \mathrm{ppm}$ CO, which is lower than the expected $10 \mathrm{ppm}$. Improvements in the light imaging system and choice of reference cell CO concentration should increase the sensitivity of the sensor by at least

American Institute of Aeronautics and Astronautics 
an order of magnitude to achieve the target sensitivity of $10 \mathrm{ppm}$ CO. The device can provide a direct output voltage proportional to the $\mathrm{CO}$ gas concentration which is available for combustion feedback control. Retrofit or new placement of these sensors on each burner will increase the efficiency (providing cost savings) as well as better meet regulatory regulations for minimizing pollutant emissions.

\section{Acknowledgments}

This project is supported by the Environmental Protection Agency (EPA) with contract number EP-D-05-040, under the Small Business Innovation Research (SBIR) program.

\section{References}

1. “Combustion Industry of the Future," Fiscal Year 2004 Anual Report, Energy Efficiency and Renewable Energy, U.S. Dept. of Energy (2005).

2. Land Combustion, http://www.landinst.com/comb.

3. Wolfrum, J., "Lasers in Combustion: From Basic Theory to Practical Devices,” Proc. Combust. Inst. 27: 1 (1998).

4. "Diode laser sensor for Combustion Control," U.S. DOE Office of Industrial Technologies, http://www.oit.doe.gov/sens cont.

5. Rothman, L.S., Barbe, A., et al., "The HITRAN Molecular Spectroscopy Database: Edition of 2000 Including updates through 2001,” J. Quant. Specrosc. Radiat. Transfer. 82: 5 (2003); L.S. Rothman, D. Jacquemart, A. Barbe et al., J.Quant. Specrosc. Radiat. Trans. (2005; in press). 